\title{
Estudo in vitro e in vivo de sondas periodontais do tipo Williams disponíveis no mercado brasileiro
}

\author{
In vitro and in vivo study of Williams-type periodontal probes available in the \\ Brazilian market
}

\section{Isabella Soares de Oliveira MATOS ${ }^{a}$ (D), Tainá Mara CARREIRA ${ }^{b} \mathbb{C}^{(0)}$, Eliane Marçon BARROSO ${ }^{b}$ (i), Gabriela Alessandra da Cruz Galhardo CAMARGO ${ }^{a, c}$ (D), Alessandra Areas e SOUZA ${ }^{a}$ (D), Elizangela Cruvinel ZUZA ${ }^{\mathrm{a}, \mathrm{c} *}$ (])}

aUFF - Universidade Federal Fluminense, ISNF - Instituto de Saúde de Nova Friburgo, Departamento de Formação Específica, Nova Friburgo, RJ, Brasil

bUNIFEB - Centro Universitário da Fundação, Faculdade de Odontologia, Departamento de Odontologia, Barretos, SP, Brasil 'UFF - Universidade Federal Fluminense, ISNF - Instituto de Saúde de Nova Friburgo, PPGO - Programa de Pósgraduação em Odontologia, Nova Friburgo, RJ, Brasil

\begin{abstract}
Como citar: Matos ISO, Carreira TM, Barroso EM, Camargo GACG, Souza AA, Zuza EC. Estudo in vitro e in vivo de sondas periodontais do tipo Williams disponíveis no mercado brasileiro. Rev Odontol UNESP. 2020;49: e20200072. https://doi.org/10.1590/1807-2577.07220
\end{abstract}

\section{Resumo}

Introdução: A sonda periodontal é a melhor ferramenta para o diagnóstico clínico da doença periodontal, porém o desenho da sonda pode influenciar nos resultados. Objetivo: Avaliar as características estruturais de sondas periodontais do tipo Williams disponíveis no mercado brasileiro e avaliar sua utilização clínica durante a sondagem periodontal. Material e método: Os grupos de sondas foram divididos em: 1) Hu-Friedy $\AA(n=15) ; 2)$ Trinity $\AA(n=15) ; 3)$ Fava $\left.{ }^{\circledR}(n=15) ; 4\right)$ Millennium $\AA(n=15)$. Foram avaliados in vitro: peso (g), diâmetro da ponta ativa ( $\mathrm{mm})$, diâmetro do cabo (mm) e milimetragem das sondas $(\mathrm{mm})$. Para o estudo in vivo, foram selecionados 10 participantes que apresentavam pelo menos quatro dentes com periodontite. As marcas de sondas foram codificadas em A, B, C e D para o cegamento do examinador. Resultado: Verificou-se um diâmetro da ponta ativa sugestivo de forma tridimensional cônica para as marcas Trinity ${ }, \mathrm{Hu}$-Friedy ${ }^{\circledR}$ e Millennium $\AA$, com conicidade crescente, enquanto a marca Fava ${ }^{\circledR}$ revelou forma tridimensional cilíndrico/paralelo. A sonda Fava ${ }^{\circledR}$ apresentou os maiores diâmetros nos terços inicial e médio, enquanto a Hu-Friedy® revelou menor diâmetro no terço inicial. Todas as sondas apresentaram milimetragem similar. No estudo clínico, verificou-se que a sonda Fava ${ }^{\circledR}$ resultou em menor média de profundidade de sondagem do que às demais. Conclusão: As sondas periodontais apresentam diferenças estruturais que devem ser consideradas durante a seleção do instrumento, sendo que as sondas cônicas apresentam resultados mais confiáveis à prática clínica, pois o desenho da sonda interfere diretamente no diagnóstico clínico da doença periodontal.

Descritores: Doenças periodontais; diagnóstico; periodontia; instrumentos odontológicos.

\begin{abstract}
Introduction: The periodontal probe is the best tool for the clinical diagnosis of periodontal disease; however the probe design can influence the results. Objective: To evaluate the structural characteristics of Williams-type periodontal probes available in the Brazilian market and evaluate their clinical use during periodontal probing. Material and method: The probe groups were divided into: 1) Hu-Friedy ${ }^{\circledR}$ $(n=15) ; 2)$ Trinity $\left.{ }^{\circledR}(n=15) ; 3\right)$ Fava $\left.{ }^{\circledR}(n=15) ; 4\right)$ Millennium $\AA(n=15)$. In vitro analyzes were performed: weight $(\mathrm{g})$, diameter of the active tip $(\mathrm{mm})$, cable diameter $(\mathrm{mm})$ and millimeter of the probes (mm). For the in vivo study, 10 participants were selected who had at least four teeth with periodontitis. The probe marks were coded in A, B, C and D for the examiner's blinding. Result: There was a diameter of the active tip suggestive of a conical three-dimensional shape for the Trinity $\AA$, Hu-Friedy® and Millennium $\AA$, with increasing taper, while the Fava ${ }^{\circledR}$ tip demonstrated a suggestive diameter of a cylindrical / parallel threedimensional shape. The Fava ${ }^{\circledR}$ probe showed the largest diameters in the initial and middle thirds, while the Hu-Friedy® revealed the smallest diameter in the initial third. All the probes presented similar
\end{abstract}


milimeter. In the clinical study, it was found that the Fava ${ }^{\circledR}$ probe resulted in a lower average of probing depth than the others. Conclusion: Periodontal probes have structural differences that must be considered during the selection of the instrument, and the conical probes present more reliable results to clinical practice, since the design of the probe directly interferes with the clinical diagnosis of periodontal disease.

Descriptors: Periodontal diseases; diagnostic; periodontics; dental instruments.

\section{INTRODUÇÃO}

A prevalência de doença periodontal em adultos tem se mostrado alta tanto em países como os Estados Unidos como no Brasil, porém formas severas da doença não atingem grande proporção da população ${ }^{1,2}$. A doença periodontal é ocasionada pelo acúmulo de biofilme ao redor dos dentes, que está diretamente relacionado à inflamação dos tecidos gengivais 3 . Durante o exame clínico, é necessário saber distinguir algumas características de como é diferenciado um tecido sadio (firme e resiliente) de um doente, em que se observam mudanças de coloração, da forma e textura dos tecidos, associados a sinais de inflamação gengival como vermelhidão, edema, sangramento à sondagem e supuração, sendo que essas condições podem ocorrer em um periodonto íntegro ou reduzido ${ }^{4,5}$.

Não só a inspeção visual das características clínicas dos tecidos gengivais é importante, porém a sondagem clínica utilizando a sonda periodontal milimetrada permanece como a melhor ferramenta a ser utilizada para o diagnóstico da doença periodontal, considerando medidas de profundidade de bolsa, presença de sangramento à sondagem, perda de inserção clínica e recessão gengival 4 .

Alguns fatores podem afetar os parâmetros periodontais avaliados, tais como presença de tecido gengival edemaciado, presença de próteses dentárias, cálculo dentário, diâmetro e/ou variações na padronização da marcação de sondas, assim como a força de sondagem exercida e erros inerentes ao operador 6 . Além do mais, a sondagem pode apresentar erros inerentes às características da própria doença, como nível de inflamação gengival, anatomia da coroa/raiz e morfologia do defeito ósseo ${ }^{7-10}$.

A sonda periodontal preconizada como ideal pela Organização Mundial da Saúde (OMS) foi desenvolvida em 1978 e segue alguns parâmetros dimensionais padronizados, evitando-se assim erros que possam ser gerados por diferenças no próprio instrumento, como ter um cabo com diâmetro de 3,5 mm; peso máximo de 4,5 g e marcações corretas e padronizadas de milimetragem. Para sondas que possuem esferas na ponta ativa, estas devem apresentar $0,5 \mathrm{~mm}$ de diâmetro. A pressão de sondagem recomendada é de 15 a 25 gramas $^{11}$.

Alguns estudos avaliaram diferentes marcas de sondas periodontais milimetradas disponíveis no mercado brasileiro ${ }^{8,11,12}$. De acordo com Cesar et $\mathrm{al}^{8}$, a sonda da Hu-Friedy® foi a que mais apresentou precisão nas medidas, aproximando-se das medidas consideradas "padrão". Como sempre há novos produtos e instrumentos odontológicos sendo lançados no mercado, verifica-se a necessidade de outros estudos que avaliem a demanda de novas marcas de instrumentos na área de periodontia, especialmente as sondas periodontais.

O objetivo deste estudo foi avaliar as características estruturais de diferentes sondas periodontais do tipo Williams disponíveis no mercado brasileiro e avaliar sua utilização clínica durante sondagem diagnóstica em periodontia.

\section{MATERIAL E MÉTODO}

O presente estudo foi dividido em duas etapas, ou seja, etapa laboratorial (avaliação in vitro) e etapa clínica (avaliação in vivo). Todas as etapas da pesquisa clínica foram conduzidas no 
ambulatório do departamento de odontologia do Instituto de Saúde de Nova Friburgo da Universidade Federal Fluminense (ISNF/UFF). 0 estudo foi submetido e aprovado pelo comitê de ética em pesquisa do ISNF, número do parecer: 3.347.468.

\section{Avaliação in vitro}

0 presente trabalho foi desenvolvido no ISNF/UFF, onde foram avaliadas 60 sondas periodontais milimetradas do tipo Williams, de diferentes marcas e diferentes lotes de fabricação, em um estudo observacional transversal.

Os grupos de estudo foram divididos com sondas periodontais milimetradas de quatro marcas, conforme segue: 1) Hu-Friedy® ( $\mathrm{n}=15$, controle) [Hu-Friedy ${ }^{\circledR}$ Mfg. Co., Chicago, EUA]; 2) Trinity ${ }^{\circledR}\left(\mathrm{n}=15\right.$, teste) [Trinity ${ }^{\circledR}$ Ind. e Com. Ltda., São Paulo, Brasil]; 3) Fava ${ }^{\circledR}(\mathrm{n}=15$, teste) [Metalúrgica Fava ${ }^{\circledR}$ Ind. e Com., São Paulo, Brasil]; 4) Millennium ${ }^{\circledR}(\mathrm{n}=15$, teste) [Golgran Ind. e Com. Ltda., São Caetano do Sul, Brasil]. Avaliaram-se as seguintes características dos instrumentos: peso (em gramas, g), diâmetro da ponta ativa no terço inicial, médio e final (em milímetros, mm), diâmetro do cabo (em milímetros, mm) e milimetragem das sondas (mm).

\section{Treinamento e Calibração}

Um único pesquisador (ISOM) foi treinado e calibrado para a realização de todas as mensurações, por um pesquisador experiente (ECZ). Para isso, foram utilizadas 12 sondas periodontais, sendo três de cada marca comercial. 0 treinamento foi realizado para todos os parâmetros (peso, diâmetro da ponta ativa, milimetragem e diâmetro do cabo). Como medidas de calibração, foram utilizadas duas mensurações principais, isto é, o diâmetro da ponta ativa e a milimetragem das sondas, que foram realizadas em dois momentos distintos, com uma semana de intervalo, sendo que a concordância entre os dados foi avaliada pelo teste de correlação intraclasse para dados contínuos, com o programa BioEstat 5.0 (Belém, PA, Brasil). A replicabilidade da correlação intraclasse (CI) se mostrou excelente tanto para a calibração do diâmetro das sondas (CI: 0,$9972 ; \mathrm{p}<0,0001$ ) quanto para a milimetragem (CI: 0,9991; $\mathrm{p}<0,0001$ ).

\section{Peso}

As sondas foram pesadas em balança digital de precisão modelo AD5000 (Marte Científica ${ }^{\circledR}$, Santa Rita do Sapucaí, Minas Gerais, Brasil), apoiada sobre uma superfície plana. Seguindo a metodologia proposta por Penteado et al. ${ }^{1}$, cada sonda foi colocada delicadamente sobre a balança e o valor anotado somente após a estabilização dos números no visor, sendo esse procedimento realizado por três vezes para cada sonda periodontal. Foram calculadas médias para cada sonda.

\section{Diâmetro da Ponta Ativa}

A metodologia seguida foi de acordo com o estudo de Penteado et al. ${ }^{11}$, dividindo-se as pontas ativas em terços: terço inicial (correspondente à marcação do segundo milímetro da ponta ativa); terço médio (correspondente à marcação do quinto milímetro da ponta ativa); e terço final (correspondente à marcação do décimo milímetro da ponta ativa) (Figura 1A). Os diâmetros das pontas ativas foram mensurados com um paquímetro digital de precisão (Mitutoyo®, Santo Amaro, São Paulo, Brasil) (Figura 1B). De cada um dos terços foram obtidas três medidas, calculando-se as médias. 

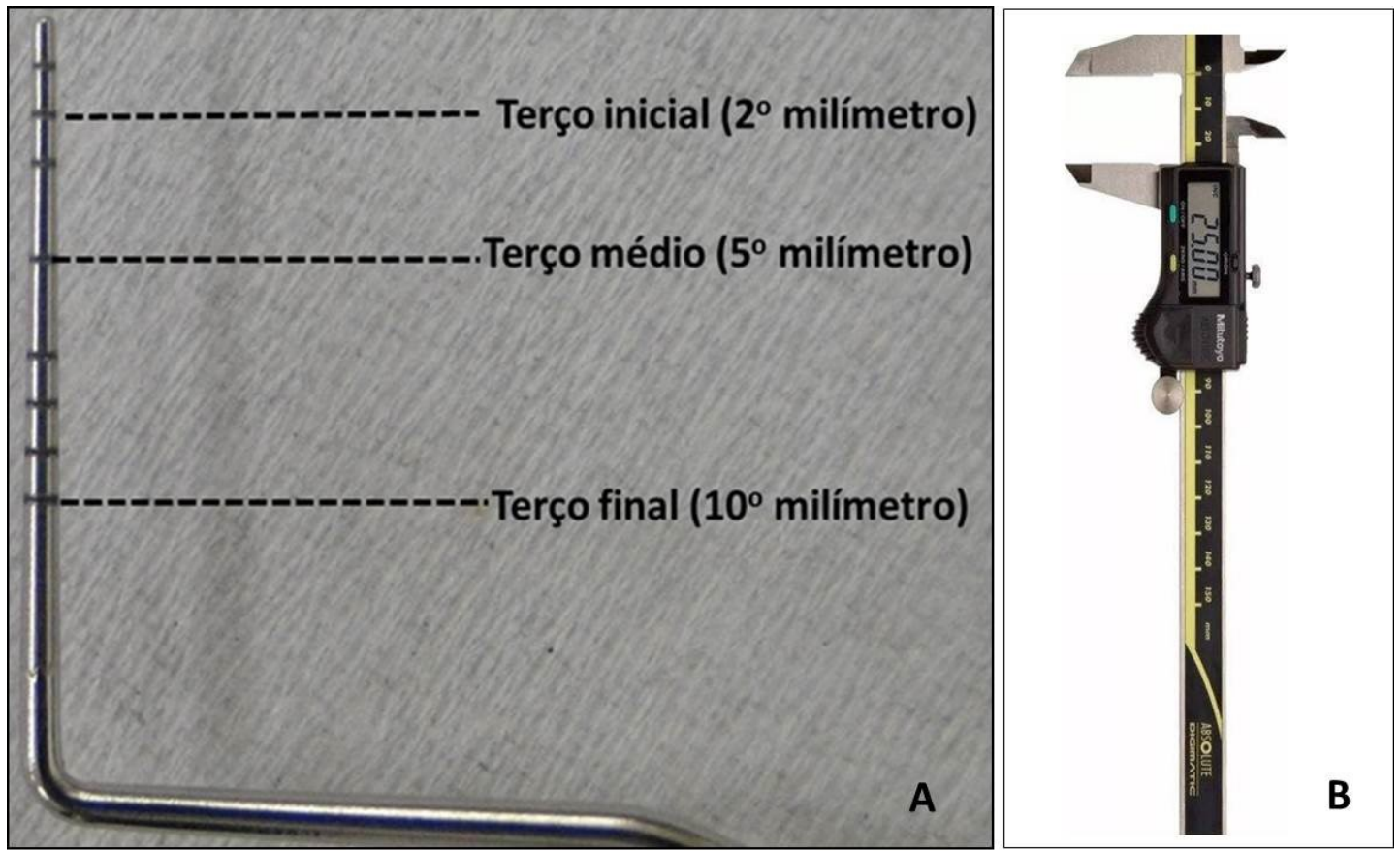

Figura 1. (A) Diâmetro da sonda periodontal do tipo Williams medido em terços inicial, médio e final; (B) Paquímetro digital de precisão.

As sondas foram posicionadas com fundo de papel branco, sendo que o examinador permaneceu com os braços apoiados em uma bancada de superfície firme e plana, para evitar deslizes e mudanças de posição que pudessem interferir com a análise. De acordo com a recomendação de Tahim et al. ${ }^{12}$, o paquímetro foi recolocado na posição inicial (zero) a cada mensuração, a fim de se evitar uma leitura viciada.

\section{Diâmetro do Cabo e Milimetragem das Sondas}

Os diâmetros dos cabos e a milimetragem dos instrumentos foram mensurados com paquímetro de precisão (Mitutoyo ${ }^{\circledR}$, Santo Amaro, São Paulo). 0 diâmetro do cabo foi medido considerando-se a porção central do instrumento. 0 padrão de marcação de milimetragem das sondas periodontais foi avaliado considerando-se a distância de 0-3 $\mathrm{mm}, 0-5 \mathrm{~mm}, 0-7 \mathrm{~mm}$ e de 0-10 mm. As medidas foram feitas por três vezes para o cálculo das médias.

\section{Avaliação in vivo}

\section{Características Gerais da População do Estudo}

Foram selecionados 10 participantes adultos, com idade $\geq 18$ anos. 0 critério de classificação de presença de periodontite foi adaptado de López et al.13, dessa forma, os indivíduos deveriam apresentar pelo menos quatro dentes com no mínimo um (1) sítio com profundidade de bolsa $\geq 5 \mathrm{~mm}$ e, concomitante, perda de inserção clínica $\geq 3 \mathrm{~mm}$.

Todos os participantes foram submetidos à anamnese e exame clínico para a identificação de condições que os elegessem ou impedissem a participação no estudo. Os indivíduos selecionados foram convidados a participar do estudo e assinaram um termo de consentimento, concordando com a sua participação voluntária no estudo, após explicação do pesquisador dos detalhes da pesquisa. 


\section{Cálculo da Amostra}

Para o cálculo do tamanho amostral foram utilizados os resultados obtidos por Barendregt et al. ${ }^{14}$, que avaliaram a profundidade de sondagem em dois momentos distintos (dia 0 e após 1 semana) e em duas sessões de sondagem em cada dia (sessão 1 e sessão 2), com a sonda manual convencional do tipo Williams (Hu-Friedy®, Chicago, IL, USA). Dessa forma, calcularam-se as diferenças das médias entre a sessão 1 e 2 em cada dia (Dia 0: 3,93-3,91 = 0,02; 1 semana após: $3,95-3,92=0,03$ ), resultando a média das diferenças de profundidade de sondagem em 0,01 . Da mesma forma, o desvio padrão das diferenças foi considerado entre o dia $0(0,04)$ e 1 semana após $(0,03)$, sendo que o resultado da diferença foi de 0,01. 0 tamanho da amostra foi calculado no programa BioEstat 5.0 (Mamirauá, Belém, Brasil), considerando o teste $t$ para duas amostras pareadas, com poder do teste de $80 \%$ e nível de significância de 0,05 . Considerando que a média das diferenças de profundidade de sondagem foi de 0,01 e que o desvio padrão das diferenças também foi de 0,01 , o resultado do tamanho da amostra foi de 10 participantes.

\section{Critérios de Inclusão}

Sujeitos adultos com faixa etária $\geq 18$ anos de idade; sujeitos com periodontite em pelo menos quatro dentes, apresentando pelo menos um (1) sítio com bolsa $\geq 5 \mathrm{~mm}$ e concomitante perda de inserção clínica $\geq 3 \mathrm{~mm}$; sujeitos que apresentem no mínimo 20 dentes avaliáveis (mínimo de cinco dentes por quadrante); sujeitos sistemicamente saudáveis.

\section{Critérios de Exclusão}

História de doenças sistêmicas, tais como diabetes mellitus, artrite reumatoide, lupus eritematoso, ou qualquer outra doença inflamatória sistêmica; mulheres grávidas e lactantes; história de uso de antibióticos e/ou anti-inflamatórios nos últimos três meses; sujeitos com hábito respiratório bucal; sujeitos com aparelhos ortodônticos; sujeitos com hábito deletério, como fumar; história da terapia periodontal não cirúrgica e/ou cirúrgica nos últimos seis meses.

\section{Calibração}

Um único examinador foi calibrado através do exame clínico de cinco participantes com pelo menos quatro dentes, mostrando no mínimo um (1) sítio com bolsa $\geq 5 \mathrm{~mm}$ e concomitante perda de inserção clínica $\geq 3 \mathrm{~mm}$. Os dentes foram sondados em duplicata com intervalo de 30 minutos $^{14}$, utilizando quatro marcas de sondas periodontais do tipo Williams (Hu-Friedy ${ }^{\circledR}$, Trinity ${ }^{\circledR}$, Fava ${ }^{\circledR}$ e Milllennium( $($ ), sendo utilizada uma em cada dente. Os dados foram analisados pelo teste de correlação intraclasse para dados contínuos pelo programa BioEstat 5.0 (Mamirauá, Belém, Brasil). A análise estatística revelou correlação intraclasse $(\mathrm{CI})$ com replicabilidade excelente $(\mathrm{p}<0,0001)$ para todas as sondas periodontais avaliadas, sendo este CI respectivamente de 0,9920

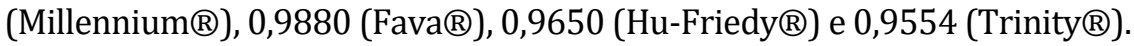

\section{Avaliação Clínica}

Para cada dente selecionado, foram sondados seis sítios (mesiovestibular, vestibular e distovestibular, distolingual, lingual e distolingual). A profundidade de sondagem (distância entre a margem gengival ao fundo da bolsa/sulco) foi avaliada clinicamente em cada sítio ${ }^{4}$. A presença/ausência de sangramento à sondagem foi verificada para considerar a presença de doença periodontal ativa ou não ${ }^{15}$. 


\section{Grupos de Estudo, Randomização e Cegamento}

Após selecionados, os participantes do estudo receberam, inicialmente, profilaxia com taça de borracha, escova de Robinson e pedra-pomes (ou pasta profilática) e instrução de higiene oral por meio da utilização da técnica de Bass modificada, para o controle do biofilme supragengival.

Os dentes selecionados foram divididos por grupos de marcas de sondas periodontais, sendo que cada dente foi sondado quatro vezes, com intervalo de 5 minutos entre sondagens ${ }^{10}$. Em cada indivíduo, cada um dos quatro dentes experimentais foi sondado, aleatoriamente, por sorteio, sendo que todas as quatro sondas foram usadas em seis sítios de cada dente, baseadas nessa randomização.

Todas as sondas periodontais do tipo Williams apresentavam milimetragem com intervalo de 1-3; 5; 7-10 mm, sendo que as marcas utilizadas foram: Hu-Friedy® [controle padrão internacional] (Hu-Friedy® Mfg. Co., Chicago, EUA), Trinity® (Trinity® Indústria e Comércio Ltda., Jaraguá, Brasil), Fava ${ }^{\circledR}$ (Metalúrgica Fava ${ }^{\circledR}$ Ind. e Com., São Paulo, Brasil) e Millennium ${ }^{\circledR}$ (Golgran Ind. e Com. Ltda., São Caetano do Sul, Brasil). As sondas foram codificadas em A, B, C e D para o cegamento do examinador. Foram utilizadas sete sondas periodontais de cada marca, sendo cada uma de lotes diferentes para evitar vieses na pesquisa.

\section{Análise Estatística}

A análise das medidas de profundidade de sondagem das diferentes sondas foi realizada considerando o sítio como unidade de medida repetida. Os resultados foram expressos em média e desvio padrão. Os dados foram testados quanto à normalidade. Foi realizada análise intergrupo por meio de testes estatísticos paramétricos (ANOVA: um critério) ou não paramétricos (Kruskal-Wallis). 0 nível de significância estatística foi estabelecido em 5\%.

\section{RESULTADO}

\section{Estudo in vitro}

A Tabela 1 demonstra diâmetro da ponta ativa sugestivo de forma tridimensional cônica para as marcas Trinity ${ }^{\circledR}$, Hu-Friedy $®$ e Millennium $®$, verificando-se uma conicidade crescente entre o terço inicial, médio e final $(\mathrm{P}<0,05)$, enquanto que a marca $\mathrm{Fava}{ }^{\circledR}$ demonstrou diâmetro sugestivo de forma tridimensional cilíndrico/paralelo, pois não houve diferenças estatisticamente significantes entre as mensurações obtidas nos diferentes terços avaliados $(p>0,05)$. A Figura 2 ilustra as conicidades encontradas entre as diferentes marcas comerciais.

Tabela 1. Média e desvio padrão (DP) dos diâmetros das sondas periodontais nos terços inicial, médio e final

Diâmetro da ponta ativa/terços

\begin{tabular}{|c|c|c|c|c|}
\hline & Inicial & Médio & Final & \\
\hline Hu-Friedy® & $0,54 \pm 0,01^{\mathrm{a}}$ & $0,64 \pm 0,009 \mathrm{~b}$ & $0,80 \pm 0,02^{c}$ & $<0,0001$ \\
\hline Trinity $®$ & $0,62 \pm 0,01$ a & $0,72 \pm 0,01 \mathrm{~b}$ & $0,86 \pm 0,01^{c}$ & $<0,0001$ \\
\hline Fava ${ }^{\circledR}$ & $0,74 \pm 0,03$ a & $0,74 \pm 0,04$ a & $0,74 \pm 0,004$ & 0,9967 \\
\hline Millennium $®$ & $0,59 \pm 0,02$ a & $0,64 \pm 0,02 \mathrm{~b}$ & $0,86 \pm 0,02^{c}$ & $<0,0001$ \\
\hline
\end{tabular}

a,b,c Letras diferentes dentro da mesma linha indicam diferença estatisticamente entre os terços avaliados (teste ANOVA: um critério, seguido de Tukey para dados paramétricos; $\mathrm{p}<0,05$ ). 


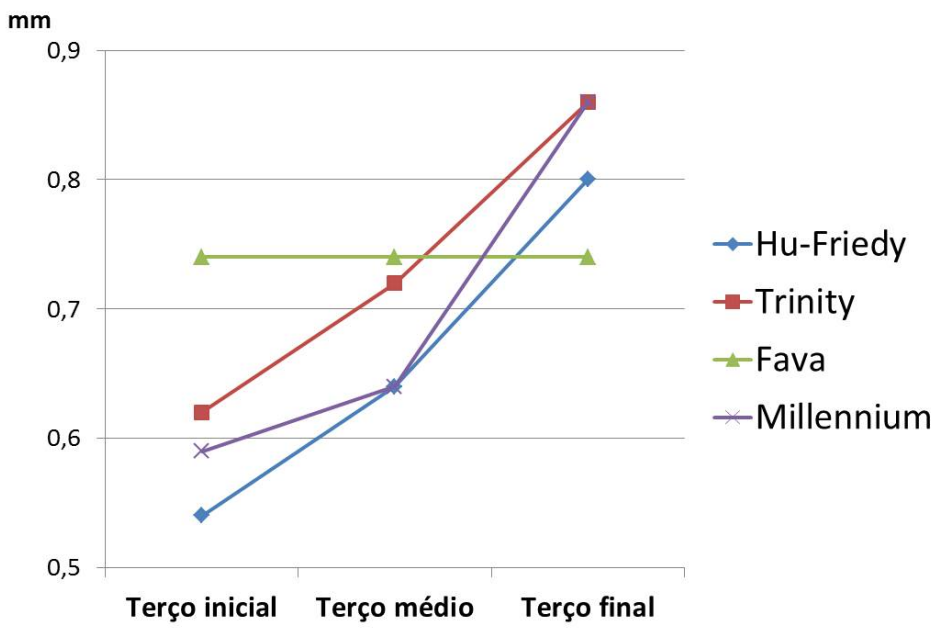

Figura 2. Gráfico ilustrando a evolução do diâmetro das diferentes marcas de sondas periodontais do tipo Williams, de acordo com os terços medidos nas marcações inicial (aos $2 \mathrm{~mm}$ ), médio (aos $5 \mathrm{~mm}$ ) e final

(aos $10 \mathrm{~mm}$ ).

Na Tabela 2 verifica-se que o peso $(20,48 \mathrm{~g})$ e o diâmetro do cabo $(9,50 \mathrm{~mm})$ foram estatisticamente maiores para a sonda da Hu-Friedy® em comparação às demais marcas $(\mathrm{P}<0,05)$. A sonda Fava ${ }^{\circledR}$ apresentou os maiores diâmetros nos terços inicial $(0,74 \mathrm{~mm})$ e médio $(0,74 \mathrm{~mm})$ $(\mathrm{P}<0,05)$. A sonda da Hu-Friedy® revelou o menor diâmetro no terço inicial $(0,54 \mathrm{~mm})$. As sondas da Millennium ${ }^{\circledR}$ e Trinity ${ }^{\circledR}$ mostraram os maiores diâmetros no terço final, apresentando ambas $0,86 \mathrm{~mm}$. Verificou-se que as sondas de todas as marcas apresentaram milimetragem similar em todos os pontos avaliados, ou seja, de 0-3 mm, 0-5 mm, 0-7 mm e 0-10 $\mathrm{mm}$ ( $\mathrm{p}>0,05)$.

Tabela 2. Média e desvio padrão (DP) das características estruturais das diferentes sondas avaliadas no estudo

\begin{tabular}{|c|c|c|c|c|c|}
\hline Parâmetros avaliados & $\begin{array}{c}\text { Hu-Friedy® } \\
\text { Média (DP) }\end{array}$ & $\begin{array}{c}\text { Trinity® Média } \\
\text { (DP) }\end{array}$ & $\begin{array}{c}\text { Fava } ® \text { Média } \\
\text { (DP) }\end{array}$ & $\begin{array}{c}\text { Millennium } \\
\text { ® Média } \\
\text { (DP) }\end{array}$ & $\begin{array}{c}\text { Valor de } \\
\text { p }\end{array}$ \\
\hline Peso $(\mathrm{g})^{*}$ & $20,48 \pm 0,13$ a & $15,37 \pm 0,0002^{b}$ & $16,95 \pm 0,05 \mathrm{~b}$ & $14,38 \pm 0,13 \mathrm{~b}$ & $<0,0001$ \\
\hline Diâmetro do cabo (mm)** & $9,50 \pm 0,01^{\mathrm{a}}$ & $7,97 \pm 0,01 \mathrm{~b}$ & $4,98 \pm 0,04^{c}$ & $7,98 \pm 0,01^{b}$ & $<0,0001$ \\
\hline Diâmetro da ponta ativa no terço inicial $(\mathrm{mm})^{* *}$ & $0,54 \pm 0,01^{\mathrm{a}}$ & $0,62 \pm 0,01 \mathrm{~b}$ & $0,74 \pm 0,03^{c}$ & $0,59 \pm 0,02 \mathrm{~b}$ & $<0,0001$ \\
\hline Diâmetro da ponta ativa no terço médio** & $0,64 \pm 0,009$ a & $0,72 \pm 0,01 \mathrm{~b}$ & $0,74 \pm 0,04 \mathrm{~b}$ & $0,64 \pm 0,02^{\mathrm{a}}$ & $<0,0001$ \\
\hline Diâmetro da ponta ativa no terço final ${ }^{* *}$ & $0,80 \pm 0,02^{\mathrm{a}}$ & $0,86 \pm 0,01 \mathrm{~b}$ & $0,74 \pm 0,04 \mathrm{c}$ & $0,86 \pm 0,02 \mathrm{~b}$ & $<0,0001$ \\
\hline Milimetragem 0-3 mm** & $2,98 \pm 0,09^{a}$ & $2,93 \pm 0,06^{\mathrm{a}}$ & $2,92 \pm 0,10^{a}$ & $2,84 \pm 0,14^{\mathrm{a}}$ & $<0,0001$ \\
\hline Milimetragem 0-5 mm** & $5,05 \pm 0,01^{\mathrm{a}}$ & $5,05 \pm 0,02^{a}$ & $5,06 \pm 0,01^{\mathrm{a}}$ & $5,05 \pm 0,01^{\mathrm{a}}$ & $<0,0001$ \\
\hline Milimetragem 0-7 mm** & $7,06 \pm 0,01^{\mathrm{a}}$ & $7,05 \pm 0,02^{a}$ & $7,06 \pm 0,01^{\mathrm{a}}$ & $7,06 \pm 0,02^{a}$ & $<0,0001$ \\
\hline Milimetragem 0-10 mm* & $10,1 \pm 0,15^{a}$ & $10,12 \pm 0,11^{\mathrm{a}}$ & $10,09 \pm 0,03^{a}$ & $10,13 \pm 0,14^{a}$ & $<0,0001$ \\
\hline
\end{tabular}

a,b,c Letras diferentes dentro da mesma linha indicam diferença estatisticamente significante entre as marcas de sondas periodontais. ${ }^{*}$ Kruskal-Wallis seguido de Dunn para dados não paramétricos $(\mathrm{p}<0,05)$. ${ }^{* *}$ ANOVA: um critério seguido de Tukey para dados paramétricos $(\mathrm{p}<0,05)$.

\section{Estudo in vivo}

Foram avaliados 10 participantes (cinco homens e cinco mulheres), com idade média de $56,6 \pm 11,85$ anos. Foram examinados quatro dentes por indivíduo, sendo sondados seis sítios por dente. Dessa forma, o exame clínico foi realizado em 40 dentes e em 240 sítios.

Os dados foram inicialmente testados com relação à normalidade pelo teste de D'AgostinoPearson, sendo considerados paramétricos. 0 teste escolhido para análise foi o ANOVA: um 
critério, considerando diferença estatisticamente significante quando o valor de $\mathrm{P}<0,05$. A comparação entre grupos de sondas pode ser visualizada na Tabela 3.

Tabela 3. Média \pm desvio padrão da profundidade de sondagem em 240 sítios, utilizando diferentes marcas comerciais de sondas periodontais disponíveis no mercado brasileiro

\begin{tabular}{cc}
\hline Marcas comerciais de sondas periodontais & Média \pm desvio-padrão \\
\hline Hu=Friedy $\AA$ & $4,01 \pm 1,44$ a \\
\hline Trinity $\AA$ & $3,73 \pm 1,45$ a \\
\hline Fava $\AA$ & $3,38 \pm 1,49 \mathrm{~b}$ \\
\hline Millennium $\AA$ & $3,75 \pm 1,47$ a \\
\hline
\end{tabular}

a,b Diferentes letras nas colunas indicam diferença estatisticamente significante entre marcas de sondas periodontais (ANOVA: um critério para dados paramétricos, $\mathrm{P}<0,05$ ).

\section{DISCUSSÃO}

0 presente estudo verificou a padronização das sondas periodontais do tipo Williams entre algumas marcas comerciais disponíveis no mercado brasileiro (Hu-Friedy®, Trinity®, Fava ${ }^{\circledR}$ e Millennium $®$ ), avaliando parâmetros como peso do instrumento, diâmetro do cabo e da ponta ativa e milimetragem. Considerando que a sondagem periodontal é o exame padrão-ouro para deteç̧ão de alterações no periodonto, entende-se que ter instrumentos calibrados seja extremamente importante no auxílio para um correto diagnóstico, uma vez que sondas periodontais não padronizadas podem interferir no diagnóstico periodontal ${ }^{16,17}$.

A sonda periodontal preconizada como ideal pela Organização Mundial da Saúde (OMS) segue alguns parâmetros dimensionais padronizados, como ter um cabo com diâmetro de 3,5 mm; peso máximo de 4,5 g e marcações corretas e padronizadas de milimetragem ${ }^{11}$. Não há recomendações da OMS para as sondas do tipo Williams; dessa forma, este estudo se baseou na metodologia adotada por Penteado et al. ${ }^{11}$, que além de estudar o peso e a distância entre as marcações das sondas periodontais, avaliou os instrumentos por terços (inicial, médio e final), o que possibilitou a avaliação da uniformidade do diâmetro ao longo da ponta ativa. Utilizou-se a sonda Williams HuFriedy ${ }^{\circledR}$ como padrão-ouro por esta apresentar marcações mais precisas e padronizadas ${ }^{8}$.

Os resultados obtidos dos pesos dos instrumentos no presente estudo foram superiores para todas as marcas avaliadas em comparação ao peso de 4,5 g, preconizado pela OMS. A padronização de um peso ideal estaria relacionada com a força de sondagem final a ser aplicada pelo examinador, de aproximadamente até $25 \mathrm{~g}$, o que teria relação direta com a profundidade de sondagem clínica obtida e com o sangramento à sondagem ${ }^{7-10}$.

As médias do diâmetro do cabo também foram superiores para todas as marcas, em comparação aos 3,5 mm, preconizado pela OMS, porém com diâmetro mais semelhante para a marca Fava ${ }^{\circledR}$ $(4,98 \mathrm{~mm})$, que apresentou cabo maciço. Por outro lado, as marcas Hu-Friedy®, Trinity ${ }^{\circledR}$ e Millennium ${ }^{\circledR}$ apresentaram diâmetros maiores, respectivamente de 9,50 mm, 7,97 mm e 7,98 mm, porém com cabos ocos, que, segundo os fabricantes, são capazes de proporcionar maior sensibilidade táctil ao operador.

Todas as marcas de sondas avaliadas apresentaram bom padrão de milimetragem, sem diferença entre marcas $(p>0,05)$. Com relação ao diâmetro da ponta ativa no terço inicial, alguns autores sugeriram que para a avaliação do nível de inserção em um tecido inflamado, uma sonda com diâmetro de ponta de $0,5 \mathrm{~mm}$ parece ser mais adequada ${ }^{18}$. Dessa forma, os nossos achados revelaram que a marca comercial que apresentou o terço inicial com média aproximada a $0,5 \mathrm{~mm}$ foi a sonda da Hu-Friedy ${ }^{\circledR}(0,54 \mathrm{~mm})$.

Uma conicidade crescente nos diferentes diâmetros (terço inicial, médio e final) foi verificada nas sondas das marcas Trinity ${ }^{\circledR}, \mathrm{Hu}$-Friedy ${ }^{\circledR}$ e Millennium ${ }^{\circledR}$, enquanto que a marca Fava ${ }^{\circledR}$ demonstrou diâmetro sugestivo de forma tridimensional cilíndrico/paralelo. 0 desenho 
cilíndrico da sonda poderia interferir com a sondagem de bolsas periodontais, especialmente profundas e estreitas, pois maior dificuldade poderia ser verificada no momento da inserção da sonda durante o exame clínico, acarretando, assim, em erro diagnóstico. Esse fato foi verificado no estudo de Bulthuis et al. ${ }^{18}$, cujos resultados mostraram que a penetração da sonda Williams de formato paralelo foi um pouco aquém $(3,96 \pm 1,71)$ para o avaliado microscopicamente, quando comparado ao formato cônico $(4,04 \pm 1,96)$. Esse fato também foi comprovado em nosso estudo, que demonstrou que a sonda de formato cilíndrico/paralelo (Fava®) foi capaz de exercer uma média de penetração menor durante a sondagem clínica em comparação ao formato cônico (Hu-Friedy ${ }^{\circledR}$, Trinity ${ }^{\circledR}$ e Millennium $\left.{ }^{\circledR}\right)$, com diferença estatisticamente significante $(\mathrm{P}<0,05)$.

Outro fator importante considerado durante o delineamento da amostra do estudo in vivo foi o diagnóstico adotado para a definição dos casos de periodontite, em que foi adaptado o critério preconizado pelo estudo de López et al. ${ }^{13}$, em que os autores definiram um paciente com periodontite quando havia $\geq 4$ dentes com $\geq 1$ sítio com profundidade de sondagem $\geq 4 \mathrm{~mm}$, associado ao nível de inserção clínico $\geq 3 \mathrm{~mm}$. No presente estudo, os mesmos critérios foram considerados, porém com uma profundidade de sondagem $\geq 5 \mathrm{~mm}$. De acordo com o estudo de Costa et al. ${ }^{19}$, diferentes critérios para o diagnóstico de periodontite têm sido descritos na literatura, resultando em grande impacto tanto na prevalência quanto na extensão da doença, sendo importante a escolha de um critério adequado para a padronização da definição de casos de periodontite para a pesquisa clínica. Esses autores ${ }^{19}$ endossam a utilização do critério utilizado por López et al. ${ }^{13}$.

Estudos clínicos bem delineados e com amostras maiores são necessários para se comprovar a aplicabilidade clínica de diferentes marcas e desenhos de sondas periodontais em tecidos periodontais sadios e com patologia instalada, a fim de melhor elucidar alguns questionamentos pertinentes à clínica odontológica.

\section{CONCLUSÃO}

Pode-se concluir que as sondas periodontais apresentam diferenças estruturais que devem ser consideradas durante a seleção do instrumento, sendo que as sondas cônicas apresentam resultados mais confiáveis à prática clínica, pois o desenho da sonda interfere diretamente no diagnóstico clínico da doença periodontal.

\section{AGRADECIMENTOS}

Agradecimento à FAPERJ (Fundação de Amparo à Pesquisa do Estado do Rio de Janeiro) por ter fornecido apoio financeiro à bolsista Isabella Soares de Oliveira Matos (número do processo: E-26/200.139/2018) durante 2 anos consecutivos.

\section{REFERÊNCIAS}

1. Brasil. Ministério da Saúde. SB Brasil 2010: Pesquisa Nacional de Saúde Bucal: resultados principais. Brasília; 2011.

2. Eke PI, Dye BA, Wei L, Slade GD, Thornton-Evans GO, Borgnakke WS, et al. Update on Prevalence of Periodontitis in adults in the United States: NHANES 2009 to 2012. J Periodontol. 2015 May;86(5):61122. http://dx.doi.org/10.1902/jop.2015.140520. PMid:25688694.

3. Loe H, Theilade E, Jensen SB. Experimental gingivitis in man. J Periodontol. 1965 May-Jun;36(3):17787. http://dx.doi.org/10.1902/jop.1965.36.3.177. PMid:14296927. 
4. Armitage GC. The complete periodontal examination. Periodontol 2000. 2004;34(1):22-33. http://dx.doi.org/10.1046/j.0906-6713.2002.003422.x. PMid:14717853.

5. Chapple ILC, Mealey BL, Van Dyke TE, Bartold PM, Dommisch H, Eickholz P, et al. Periodontal health and gingival diseases and conditions on an intact and a reduced periodontium: consensus report of workgroup 1 of the 2017 World Workshop on the Classification of Periodontal and Peri-Implant Diseases and Conditions. J Clin Periodontol. 2018 Jun;45(Suppl 20):S68-77. http://dx.doi.org/10.1111/jcpe.12940. PMid:29926499.

6. Andrade R, Espinoza M, Gómez EM, Rolando Espinoza J, Cruz E. Intra- and inter-examiner reproducibility of manual probing depth. Braz Oral Res. 2012 Jan-Feb;26(1):57-63. http://dx.doi.org/10.1590/S1806-83242012000100010. PMid:22344339.

7. Magalhães D, Campoli MAO, Teixeira CPR. Avaliação da precisão de sondagem periodontal. ROBRAC. 1993;3(7):4-6.

8. Cesar JB No, Nogueira GR Fo, Tramontina VA, Sallum EA, Nociti FH Jr, Sallum AW. Millimeter marks and probe tip diameter standardisation from commercially available periodontal probes: a comparative study. J Int Acad Periodontol. 2001 Jul;3(3):57-60. PMid:12666942.

9. Rocha EF, Campanelli V, Crivelenti LAM, Joaquim AMC. Variability of the probing depth between conventional and controlled-force periodontal probes. Rev Salusvita. 2003;22(2):219-27.

10. Pinto FM, Gusmão ES, Souza EHA, Silveira RCJ. Sondagem clínica do sulco gengival: avaliação da sua variabilidade e reprodutibilidade. RGO (Porto Alegre). 2006 Jan-Mar;54(1):39-42.

11. Penteado LAM, Awabdi TWM, Auto VC, Rodrigues EDO, Oliveira DP, Santos NB. Avaliação da padronização das sondas periodontals utilizadas em uma instituição de ensino superior de Maceió-AL. Int J Dent. 2010;9(3):120-7.

12. Tahim CM, Barbosa CS, Mota OML, Pereira SLS, Lima DLF, Carlos MX. Avaliação da padronização de sondas periodontais tipo Williams. Revista Periodontia. 2007 Set;17(3):86-9.

13. López NJ, Smith PC, Gutierrez J. Periodontal therapy may reduce the risk of preterm low birth weight in women with peridotal disease: a randomized controlled trial. J Periodontol. 2002 Aug;73(8):911-24. http://dx.doi.org/10.1902/jop.2002.73.8.911. PMid:12211502.

14. Barendregt DS, Van Der Velden U, Timmerman MF, Van Der Weijden GA. Comparison of two automated periodontal probes and two probes with a conventional readout in periodontal maintenance patients. J Clin Periodontol. 2006 Apr;33(4):276-82. http://dx.doi.org/10.1111/j.1600051X.2006.00900.x. PMid:16553636.

15. Ainamo J, Bay I. Problems and proposals for recording gingivitis and plaque. Int Dent J. 1975 Dec;25(4):229-35. PMid:1058834.

16. Research, Science and Therapy Committee. Position paper: diagnosis of periodontal diseases. J Periodontol. 2003 Aug;74(8):1237-47. http://dx.doi.org/10.1902/jop.2003.74.8.1237. PMid:29539063.

17. Highfield J. Diagnosis and classification of periodontal disease. Aust Dent J. 2009 Sep;54(Suppl 1):S1126. http://dx.doi.org/10.1111/j.1834-7819.2009.01140.x. PMid:19737262.

18. Bulthuis HM, Barendregt DS, Timmerman MF, Loos BG, Van Der Velden U. Probe penetration in relation to the connective tissue attachment level: Influence of tine shape and probing force. J Clin Periodontol. 1998 May;25(5):417-23. http://dx.doi.org/10.1111/j.1600-051X.1998.tb02465.x. PMid:9650880.

19. Costa FO, Guimarães AN, Cota LOM, Pataro AL, Segundo TK, Cortelli SC, et al. Impact of different periodontitis case definitions on periodontal research. J Oral Sci. 2009 Jun;51(2):199-206. http://dx.doi.org/10.2334/josnusd.51.199. PMid:19550087. 


\section{CONFLITOS DE INTERESSE}

Os autores declaram não haver conflitos de interesse.

\section{*AUTOR PARA CORRESPONDÊNCIA}

Elizangela Cruvinel Zuza, UFF - Universidade Federal Fluminense, Rua Dr. Silvio Henrique Braune, 22, Centro, 28625-650 Nova Friburgo - RJ, Brasil, e-mail: elizangelazuza@yahoo.com.br

Recebido: Outubro 31, 2020

Aprovado: Novembro 18, 2020 